\title{
The Relationship of Psychiatric Symptoms with Performance-Based and Self-Reported Cognitive Function After Ischemic Stroke
}

\author{
Elisabeth Kliem ${ }^{1 *}$, Elise Gjestad ${ }^{1,2}$ (1) , Truls Ryum ${ }^{1}$, Alexander Olsen ${ }^{1,3}$, Bente Thommessen ${ }^{4}$, Bent Indredavik ${ }^{5,6}$, \\ Linas Bieliauskas ${ }^{7}$, Paulina Due-Tønnessen ${ }^{8}$, Tormod Fladby ${ }^{4,9}$ and Ramune Grambaite ${ }^{1,10}$ \\ ${ }^{1}$ Department of Psychology, Norwegian Institute of Science and Technology, Trondheim, Norway \\ ${ }^{2}$ Clinic of Medicine, St. Olav's Hospital, Trondheim University Hospital, Norway \\ ${ }^{3}$ Department of Physical Medicine and Rehabilitation, St. Olav's Hospital, Trondheim University Hospital, Trondheim, Norway \\ ${ }^{4}$ Department of Neurology, Akershus University Hospital, Lørenskog, Norway \\ ${ }^{5}$ Department of Neuromedicine and Movement Science, Norwegian Institute of Science and Technology, Trondheim, Norway \\ ${ }^{6}$ Department of Medicine, Stroke Unit, St. Olav's Hospital, Trondheim University Hospital, Trondheim, Norway \\ ${ }^{7}$ Neuropsychology Section of Psychiatry, Michigan Medicine, Ann Arbor, Michigan, USA \\ ${ }^{8}$ Department of Radiology and Nuclear Medicine, Oslo University Hospital, Oslo, Norway \\ ${ }^{9}$ Institute of Clinical Medicine, University of Oslo, Oslo, Norway \\ ${ }^{10}$ Health Services Research Unit (HØKH), Akershus University Hospital, Lørenskog, Norway
}

(Received July 17, 2020; Final Revision November 25, 2020; Accepted January 17, 2021; First Published Online March 31, 2021)

\begin{abstract}
Objective: Findings on the relationship of psychiatric symptoms with performance-based and self-reported cognitive function post-stroke are inconclusive. We aimed to (1) study the relation of depression and anxiety to performancebased cognitive function and (2) explore a broader spectrum of psychiatric symptoms and their association with performance-based versus self-reported cognitive function. Method: Individuals with supratentorial ischemic stroke performed neuropsychological examination 3 months after stroke. For primary analyses, composite scores for memory and attention/executive function were calculated based on selected neuropsychological tests, and the Hospital Anxiety and Depression Scale (HADS) was used. Psychiatric symptoms and self-reported cognitive function for secondary aims were assessed using the Symptom-Checklist-90 - Revised (SCL-90-R). Results: In a sample of 86 patients [mean ( $M$ ) age: $64.6 \pm 9.2$; Mini-Mental State Examination (MMSE), 3-7 days post-stroke: $M=28.4 \pm 1.7$; National Institutes of Health Stroke Scale (NIHSS) after 3 months: $M=0.7 \pm 1.6$ ] depressive symptoms (HADS) were associated with poorer memory performance after controlling for age, sex, and education $(\mathrm{p} \leq .01)$. In a subsample $(n=41$; Age:

$M=65.7 \pm 8.1$; MMSE: $M=28.4 \pm 1.8$; NIHSS: $M=1.0 \pm 1.9$ ), symptoms of phobic anxiety (SCL-90-R) were associated with poorer performance-based memory and attention/executive function, and symptoms of anxiety (SCL-90-R) with lower attention/executive function. Higher levels of self-reported cognitive difficulties were associated with higher scores in all psychiatric domains $(\mathrm{p} \leq .05)$. Conclusion: Even in relatively well-functioning stroke patients, depressive symptoms are associated with poorer memory. The results also suggest that various psychiatric symptoms are more related to self-reported rather than to performance-based cognitive function. Screening for self-reported cognitive difficulties may not only help to identify patients with cognitive impairment, but also those who need psychological treatment.
\end{abstract}

Keywords: Ischemic stroke, Memory, Attention/executive function, Psychiatric symptoms, Subjective cognitive function, Neuropsychological assessment

\section{INTRODUCTION}

Stroke affects approximately 14 million people per year worldwide (Johnson et al., 2019), many of whom will experience cognitive impairment and psychiatric symptoms after

*Correspondence and reprint requests to: E. Kliem, Department of Psychology, NTNU, 7491 Trondheim, Norway. E-mail: elisabeth.kliem@ ntnu.no the event, resulting in a major burden on patients, families, and the society.

In total, about one-third to half of stroke survivors develop psychiatric symptoms (Ferro, Caeiro, \& Figueira, 2016). These symptoms often stay undetected (Ferro et al., 2016) and patients' clinical needs remain unmet (McKevitt et al., 2011). If untreated, psychiatric symptoms are likely to have a limiting effect on rehabilitation and lead to higher rates of 
disability and mortality (Williams, Ghose, \& Swindle, 2004). Among psychiatric post-stroke sequelae, symptoms like anxiety, depression, and apathy are particularly common (Hackett, Kohler, O’Brien, \& Mead, 2014). Such sequelae may be caused by brain damage: Frontal/anterior and basal ganglia strokes, and both large and multiple strokes have been related to depression in the post-acute phase (Medeiros, Roy, Kontos, \& Beach, 2020). Several pathophysiological processes have been described, including abnormal neurotrophic activity, and decreased monoamine levels, where ischemic strokes are thought to disturb neural pathways from the brainstem to the cerebral cortex leading to low monoamine levels in limbic structures of frontal-temporal regions and basal ganglia (Loubinoux et al., 2012). Additionally, life changes post-stroke, such as the loss of autonomy and disability may contribute to psychiatric symptoms (Medeiros et al., 2020). Even after mild stroke, patients still experience a negative long-term effect on their lives, which is associated with elevated fatigue and emotional symptoms (Carlsson, Moller, \& Blomstrand, 2003; Terrill, Schwartz, \& Belagaje, 2018). Approximately one-third of stroke patients experience depression after 2-3 years despite excellent physical recoveries (Kapoor et al., 2017).

Besides psychiatric symptoms, cognitive impairment is common post-stroke (Sun, Tan, \& Yu, 2014). Impaired cognition has been found to be an important predictor of stroke recovery, leading to substantial functional difficulties (Zinn et al., 2004) and hindering successful rehabilitation (Skidmore et al., 2010). Even in cases with excellent physical recovery after stroke, more than half of the patients may still experience cognitive impairment 1 year (Ihle-Hansen et al., 2011) and several years after stroke (Kapoor et al., 2017).

Cognitive function can be evaluated using both performance-based and self-report measures. Studies using performance-based measures have shown that different cognitive domains are affected depending on both the location and the size of the stroke. Even though there is no distinct performance-based cognitive profile post-stroke, deficits in processing speed and executive function seem to occur most frequently, and across different types of stroke pathology and location (Cumming, Marshall, \& Lazar, 2012). Less details are known about subjective cognitive function post-stroke, despite $28.6 \%-92.0 \%$ of patients reporting cognitive difficulties (van Rijsbergen, Mark, de Kort, \& Sitskoorn, 2014). Cognitive difficulties are often reported by patients early after stroke (Nijsse et al., 2017) and remain relatively stable from 3 to 12 months post-stroke (van Rijsbergen, Mark, Kop, de Kort, \& Sitskoorn, 2020). Of importance, poorer selfreported cognitive function is associated with lower quality of life post-stroke, and may predict future performance-based cognitive difficulties (van Rijsbergen et al., 2014).

Traditionally, research has focused on the relation of depression and anxiety with cognitive function. Here, depression has typically been associated with poorer performancebased cognitive function post-stroke (Hackett et al., 2014; Robinson \& Jorge, 2016). This relationship between depressive symptoms and poorer cognitive function was also found in patients with minor stroke who have relatively good general outcomes and a promising long-term prognosis (Morsund et al., 2019). The association between anxiety and performance-based cognitive function seems to be less clear, and contradictory findings exist (Grosdemange et al., 2015; Morsund et al., 2019). In studies using self-report measures of cognitive function, higher levels of self-reported cognitive difficulties are commonly related to higher levels of depression post-stroke (van Rijsbergen et al., 2014). Additionally, anxiety, fatigue, and general psychological distress seem to be associated with reports of poorer cognition (van Rijsbergen et al., 2014).

With most studies focusing on the role of depression and anxiety, little is known about a broader spectrum of psychiatric symptoms (e.g., obsessive-compulsive symptoms or somatization) and their relation to cognitive function. For performance-based cognitive function, preliminary evidence suggests that general psychiatric distress, anxiety, depression, and insufficiency of thinking and acting predict cognitive decline 1-6 months post-stroke (Rasquin, Lodder, \& Verhey, 2005), but research in this area is sparse.

In patients with mild stroke and relatively good outcome post-stroke, psychiatric and cognitive difficulties may remain undetected in general clinical practice, but still hinder full participation in rehabilitation services and impact quality of life. Hence, understanding the association between these post-stroke sequelae is crucial in order to determine patients in need of further treatment. The aim of this study was, therefore, twofold (Figure 1). First, we studied the relationship between performance-based cognitive function and symptoms of depression and anxiety in relatively well-functioning stroke patients 3 months post-stroke $(n=86)$. We hypothesized that higher levels of anxiety and depression would be associated with poorer attention/executive function and memory. Second, we evaluated a broader spectrum of psychiatric symptoms and their association to self-reported and performance-based cognitive function in a subsample $(n=41)$.

\section{METHOD}

\section{Participants}

This study used data from a project conducted between April 2007 and April 2010 at the Stroke Unit, Department of Neurology, Akershus University Hospital (Ahus), Norway. Participants were consecutively recruited from the Stroke Unit at Ahus, and patients with supratentorial stroke, aged between 40 and 79 years, Mini-Mental State Examination (MMSE) (Folstein et al., 1975) score $\geq 23$, and no significant symptoms of visual and/or auditory neglect that would have influenced test results were included. Patients with previous hospitalizations due to stroke or with a history of other neurologic and/or psychiatric disorders (self-reported or documented in patients' journals), as well as patients with speech/language difficulties as indicated during the MMSE and/or clinical interview were excluded. Diagnosis and type of stroke were determined by neurologists, based on clinical 


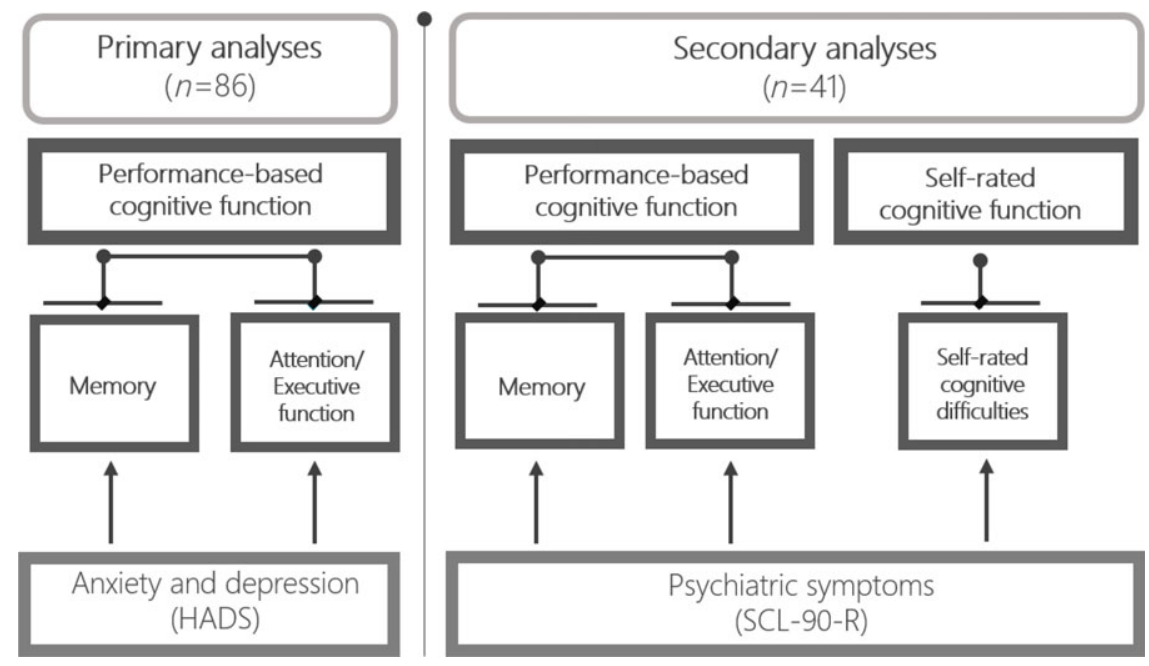

Figure 1. Overview of included variables and analyses

symptoms and supported by imaging data [computed tomography for all patients, and magnetic resonance imaging (MRI) when available].

Initially, 97 stroke patients who agreed to neuropsychological examination, MRI, and cerebrospinal fluid examination were included. Eighty-six participants remained in the study after 3 months and were included in the primary analyses. Dropout was mainly due to illness, and patients who experienced new strokes between the testing phases were excluded. In subsample analyses for secondary aims, only those patients $(n=41)$ who completed a questionnaire assessing psychiatric symptoms (the Symptom-Checklist90 - Revised; SCL-90-R) at the 3-month follow-up were included. The full sample is also described in a previous study (Selnes et al., 2015).

Participants' consent was obtained according to the Declaration of Helsinki. The study was approved by the Regional Committees for Medical and Health Research Ethics in Norway and carried out in accordance with the Norwegian Health and Research Act.

\section{Measures}

Data included in the analyses were collected while the participants were monitored or received follow-up at the Department of Neurology. All tests were performed in Norwegian by the same neuropsychologist (R.G.).

\section{Functional Measures}

The National Institutes of Health Stroke Scale (NIHSS) was used to quantify neurologic impairment 1 day and 3 months post-stroke. The NIHSS is composed of 11 items, each scoring a specific ability, e.g., language or motoric function. Scores range from 0 (=normal function) to 2-4 (=different levels of impairment). The highest possible score for noncomatose patients is 42 .
The Barthel Index (Mahoney \& Barthel, 1965) was used to measure performance in activities of daily living (ADL) 3-7 days, and 3 months post-stroke. Ten items describing basic ADL function, e.g., toilet use or dressing, assess the degree of assistance required. Higher scores indicate more independence. The total score ranges from 0 to 100 .

\section{Psychiatric Symptoms}

The SCL-90-R (Derogatis, 1992) measured overall psychopathological distress and nine psychiatric dimensions 3 months post-stroke: Depression, anxiety, phobic anxiety, somatization, interpersonal sensitivity, obsessive-compulsive symptoms, hostility, paranoid ideation, and psychoticism. Items are rated on a 5-point scale from "not at all" (0) to "extremely" (4). Subscale scores are obtained by summing up raw scores of all items belonging to a given subscale and dividing this value by the number of items. For the obsessive-compulsive-subscale, the two items measuring subjective cognitive difficulties were removed as we assumed that subjective cognitive difficulties may, independently from other obsessive-compulsive symptoms, influence performancebased cognitive function. Raw scores were converted into $T$ scores by using age- and sex-keyed norms from the manual to identify the prevalence of clinical symptoms $(T$ score $\geq 63)$ (Derogatis, 1992). The SCL-90-R was found to be a valid measure for general psychopathology in a Norwegian sample (Carrozzino et al., 2016).

We also administered the Hospital Anxiety and Depression Scale (HADS) (Zigmond \& Snaith, 1983), which consists of two subscales evaluating depression and anxiety. HADS has been validated in stroke patients (Aben, Verhey, Lousberg, Lodder, \& Honig, 2002), and the Norwegian version has shown to be a valid screening tool in a Norwegian stroke population (Fure, Wyller, Engedal, \& Thommessen, 2006). Items are rated on a 4-point scale from "not at all" (0) to "most of the time" (3). A score of $\geq 8$ on either subscale 
indicates a possible clinical problem (Leiknes, Dalsbø, \& Siqveland, 2016).

\section{Performance-Based Cognitive Function}

All neuropsychological tests were performed 3 months poststroke, except MMSE, which was assessed 3-7 days post-stroke. General cognitive ability was tested using the Vocabulary and Matrix Reasoning subtests from the Wechsler Abbreviated Scale of Intelligence (WASI; Wechsler, 1999).

Selected neuropsychological tests were used to form two composite scores: One for memory and one for attention, executive function, and processing speed. To enhance readability, the term attention/executive function was used for the latter. Composite variables were calculated based on $z$ scores (Mean $=0 ; S D=1$ ), following a common procedure when developing composite scores of neuropsychological tests (Boone, Miller, Swartz, Lu, \& Lee, 2003; Gibbons et al., 2012). $Z$ scores were internally standardized by first subtracting the mean test score of all participants from the raw score in a given test, and then dividing this value by the sample $S D$ (Song et al., 2013). When lower scores indicate better performance (TMT-A/B, CWIT-3/4), raw scores were multiplied by -1 , so that higher scores represented better performance in all tests. To get the final composite scores, individual $z$ scores were summed and divided by the number of included tests. Higher composite scores indicate better performance.

The following tests were included in the memory composite score:

1. The immediate and delayed recall trial of the Rey Auditory Verbal Learning Test ("RAVLT-1", "RAVLT-2") (Schmidt, 1996) were used to assess verbal memory. The immediate recall trial measures free recall ability of a 15 -item wordlist immediately after five learning trials and an interference list, whereas the delayed trial measures recall ability $30 \mathrm{~min}$ after the last learning trial. The score is the number of correctly recalled words. Higher scores indicate better performance.

2. The immediate and delayed recall trial ("REY-1", "REY-2") of the Rey Complex Figure Test (Meyers \& Meyers, 1995) were used to assess visuospatial memory. The immediate recall trial is administered $3 \mathrm{~min}$ after a copy trial, and delayed recall is administered after $30 \mathrm{~min}$. The score is calculated based on the accuracy of reproducing the figure. Higher scores indicate better performance.

The following tests were included in the attention/ executive function composite score:

1. Trail Making Tests A and B ("TMT-A", "TMT-B") (Reitan \& Wolfson, 1985). TMT covers visual scanning, processing speed, and divided attention (Lezak, Howieson, \& Loring, 2004). The score is the number of seconds spent on each task. Lower scores indicate better performance.

2. Digit Symbol-Coding subtest ("Coding") of WAIS-III (Wechsler, 2003) is a timed measure (120 s) of processing speed, attention, learning, and information organization. The score is calculated by counting correctly drawn symbols. Higher scores indicate better performance.
3. Color-Word Interference Test conditions 3 and 4 ("CWIT-3", "CWIT-4") of the Delis-Kaplan Executive Function System (D-KEFS) battery (Delis, Kaplan, \& Kramer, 2001) were used. CWIT-3 captures response inhibition and CWIT-4 measures inhibition and cognitive flexibility. The score is the number of seconds spent on each task. Lower scores indicate better performance.

\section{Self-Reported Cognitive Difficulties}

Two items of the SCL-90-R obsessive-compulsive subscale addressing memory and concentration difficulties, rated on a 5-point scale from "not at all" (0) to "extremely" (4), were used to measure self-reported cognitive difficulties 3 months post-stroke.

A composite score was formed using $z$ scores. Raw scores were transformed into $z$ scores using the same procedure described above (internal standardization). $Z$ scores were summed and divided by two (i.e., the number of items included in the composite variable). Higher composite scores indicate more self-reported cognitive difficulties.

\section{Statistics}

The Statistical Package for Social Sciences, version 25 (IBM Corp, 2017), was used. To compare characteristics of patients that filled out the SCL-90-R with those who did not, the independent samples $t$ test and the $\chi^{2}$ test were used. Associations between self-reported cognitive difficulties and test performance were studied using Pearson correlation.

Relations between psychiatric symptoms and cognitive function were analyzed using multiple linear regressions. In primary analyses $(n=86)$, we investigated the relation between HADS and performance-based cognitive function. In subsample analyses $(n=41)$ for secondary aims, we explored the relations of a broader spectrum of psychiatric symptoms to performance-based and self-reported cognitive function (Figure 1). Based on literature, we included age, sex, and education as predictors into all regression models. Predictors were entered simultaneously.

An examination of the data showed no violation of assumptions for regression analysis. For some cases, bootstrapping was used due to tendencies of the skewed distribution of residuals.

For our primary analyses, we chose the significance level $\mathrm{p} \leq .01$ to adjust for multiple testing. Results of the subsample analyses for secondary aims were not adjusted for multiple testing, and the standard significance level $\mathrm{p} \leq .05$ was used. To estimate effect sizes, Cohen's $d$ was used for continuous variables, where $d \geq .20$ indicates a small effect, $d \geq .50$ a medium, and $d \geq .80$ a large effect (Cohen, 1988). For categorical variables, Cramer's phi $\left(\phi_{c}\right)$ was used with $\phi_{c}=.10$ indicating a small effect, $\phi_{c}=.30$ a medium, and $\phi_{c}=.50$ a large effect when the number of degrees of freedom $=1$ (Cohen, 1988). 
Table 1. Patient characteristics

\begin{tabular}{|c|c|c|c|c|c|c|c|}
\hline & \multirow{2}{*}{ 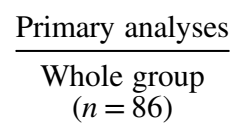 } & \multicolumn{2}{|c|}{ Subsample analyses } & \multirow[b]{2}{*}{$\mathrm{t} / \chi^{2}$} & \multirow[b]{2}{*}{$d f$} & \multirow[b]{2}{*}{$p$} & \multirow[b]{2}{*}{$d / \varphi_{\mathrm{c}}$} \\
\hline & & $\begin{array}{l}\text { SCL-90-R group } \\
\quad(n=41)\end{array}$ & $\begin{array}{c}\text { Excluded patients }^{1} \\
\quad(n=45)\end{array}$ & & & & \\
\hline Age $41-79, M(S D), \mathrm{y}$ & $64.6(9.2)$ & $65.7(8.1)$ & $63.6(10.2)$ & -1.1 & 84 & .29 & .23 \\
\hline Education $7-17, M(S D), \mathrm{y}$ & $11.0(2.9)$ & $11.0(2.9)$ & $11.0(3.0)$ & 0.01 & 84 & $>.99$ & $<.01$ \\
\hline Sex, $n$ male $(\%)$ & $59(68.6 \%)$ & $33(80.5 \%)$ & $26(57.8 \%)$ & 5.1 & 1 & $.02 *$ & .24 \\
\hline Handedness, $n$ right (\%) & $77(89.5 \%)$ & $35(85.4 \%)$ & $42(93.3 \%)$ & 0.9 & 1 & .38 & .10 \\
\hline Stroke type, $n$ lacunar (\%) & $49(57.0 \%)$ & $24(58.5 \%)$ & $25(55.6 \%)$ & 0.02 & 1 & .87 & .02 \\
\hline NIHSS, baseline $^{2}, M(S D)$ & $3.6(3.4)$ & $3.6(3.4)$ & $3.6(3.4)$ & 0.1 & 84 & .96 & .01 \\
\hline NIHSS, 3 months $^{2}, M(S D)$ & $0.7(1.6)$ & $1.0(1.9)$ & $0.5(1.1)$ & -1.4 & 84 & .17 & .30 \\
\hline Barthel, baseline, $M(S D)$ & $92.2(18.3)$ & $90.5(19.8)$ & $93.7(16.9)$ & 0.8 & 84 & .42 & .17 \\
\hline Barthel, 3 months, $M(S D)$ & $97.7(7.5)$ & $97.4(7.8)$ & $97.9(7.3)$ & 0.3 & 84 & .78 & .06 \\
\hline HADS-A, 3 months ${ }^{2}, M(S D)$ & $4.4(3.8)$ & $3.6(3.5)$ & $5.1(4.0)$ & 1.9 & 84 & .07 & .40 \\
\hline HADS-D, 3 months $^{2}, M(S D)$ & $3.6(3.3)$ & $3.6(2.9)$ & $3.6(3.7)$ & 0.8 & 84 & .94 & .02 \\
\hline Vocabulary, 3 months ${ }^{3}, M(S D)$ & $53.7(9.5)$ & $54.0(10.0)$ & $53.4(9.2)$ & -0.3 & 84 & .78 & .06 \\
\hline Matrix Reasoning, 3 months ${ }^{3}, M(S D)$ & $54.6(9.6)$ & $56.9(8.7)$ & $52.5(10.0)$ & -2.1 & 84 & $.04 *$ & .46 \\
\hline MMSE, baseline $e^{4}, M(S D)$ & $28.4(1.7)$ & $28.4(1.8)$ & $28.4(1.8)$ & 0.2 & 75 & .88 & .04 \\
\hline
\end{tabular}

*Significant group difference at $\mathrm{p} \leq .05$. Raw scores are presented (if not noted otherwise). ${ }^{1}$ Excluded patients $=$ Patients who did not answer the SCL-90-R and thus were not used in the subsample analyses. ${ }^{2}$ Higher values indicate higher impairment/higher levels of symptoms. ${ }^{3} T$ scores presented (age- and sex-keyed norms from the manual used). ${ }^{4}$ Range: $0-30$. Baseline $=$ Within the first week after stroke.

\section{RESULTS}

\section{Patient Characteristics}

Patient and stroke characteristics are shown in Table 1 and Supplementary Table 1, respectively. All patients were Caucasian with a Scandinavian language as their mother tongue, and all received treatment as usual despite their participation in the study. None of the patients received a specialized cognitive rehabilitation during the first 3 months post-stroke. Men and women did not significantly differ in age in the full sample (mean $(M)_{\text {women }}=65.7 \pm 8.0 ; M_{\text {men }}=$ $64.1 \pm 9.9 ; \mathrm{p}=.45)$, nor in the subsample $\left(M_{\mathrm{women}}=67.4 \pm\right.$ 5.7; $M_{\text {men }}=65.3 \pm 8.7 ; \mathrm{p}=.53$ ). To investigate if patients included in subsample analyses significantly differed from those who did not respond to the SCL-90-R, possibly leading to a bias, we compared characteristics of these two subsamples. Patients who were included in subsample analyses significantly differed from patients that were not included only in sex, with fewer women in the sample used for subsample analyses $(19.5 \%$ vs. $42.2 \%$ ), and in one measure of general cognitive function (Matrix Reasoning), with patients included in subsample analyses having higher scores. Raw scores for performance-based cognitive function are displayed in Table 2.

\section{Primary Analyses: Relations of Symptoms of Anxiety and Depression to Performance-Based Cognitive Function}

In the full sample $(n=86), 16$ patients $(18.6 \%)$ had HADS-A scores above cutoff, and 11 (12.8\%) had HADS-D scores above cutoff.
Table 2. Raw scores for performance-based cognitive function in both samples

\begin{tabular}{|c|c|c|c|c|}
\hline & \multicolumn{2}{|c|}{$\begin{array}{l}\text { Primary analyses } \\
(n=86)\end{array}$} & \multicolumn{2}{|c|}{$\begin{array}{c}\text { Subsample } \\
\text { analyses }(n=41)\end{array}$} \\
\hline & Mean & $S D$ & Mean & $S D$ \\
\hline \multicolumn{5}{|c|}{ Attention/Executive function } \\
\hline TMT-A & 48.03 & 2.15 & 51.24 & 3.64 \\
\hline TMT-B & 118.81 & 5.80 & 123.32 & 7.89 \\
\hline CWIT-3 & 78.42 & 3.30 & 78.02 & 4.40 \\
\hline CWIT-4 & 82.12 & 2.80 & 84.95 & 3.74 \\
\hline Coding & 44.08 & 1.63 & 42.05 & 2.26 \\
\hline \multicolumn{5}{|l|}{ Memory } \\
\hline RAVLT-1 & 8.70 & 0.35 & 8.37 & 0.54 \\
\hline RAVLT-2 & 8.23 & 0.39 & 7.61 & 0.60 \\
\hline REY-1 & 13.73 & 0.67 & 13.55 & 1.01 \\
\hline REY-2 & 13.22 & 0.72 & 13.50 & 1.07 \\
\hline
\end{tabular}

All attention/executive function tests are measured in time spent on the tasks (seconds). For RAVLT-1 and -2, the number of correctly recalled words is displayed. For REY-1 and -2, raw scores based on the accuracy of reproducing the figure are displayed.

Results of all primary analyses are displayed in Table 3. There was a significant negative association between HADS-D and memory, but not between HADS-D and attention/executive function. Thus, stroke patients with higher levels of depressive symptoms had significantly lower scores on memory tests, but not on tests measuring attention/ executive function. HADS-A was neither significantly related to memory nor to attention/executive function. Age was a significant predictor $(\mathrm{p} \leq .01)$ in all analyses, with older participants having poorer results. Women achieved 
Table 3. Primary analyses $(n=86)$ : associations between anxiety and depression and performance-based cognitive function $(n=86)$

\begin{tabular}{|c|c|c|c|c|c|c|c|c|c|c|}
\hline & \multicolumn{5}{|c|}{ Memory } & \multicolumn{5}{|c|}{ Attention/Executive function } \\
\hline & \multirow[b]{2}{*}{$B$} & \multirow[b]{2}{*}{$p$} & \multicolumn{2}{|c|}{$95 \% \mathrm{CI}$} & \multirow[b]{2}{*}{$R^{2}$} & \multirow[b]{2}{*}{$B$} & \multirow[b]{2}{*}{$p$} & \multicolumn{2}{|c|}{$95 \% \mathrm{CI}$} & \multirow[b]{2}{*}{$R^{2}$} \\
\hline & & & Lower & Upper & & & & Lower & Upper & \\
\hline HADS-D & -0.07 & $.01 *$ & -0.12 & -0.02 & .24 & -0.05 & .04 & -0.09 & 0.00 & .34 \\
\hline HADS-A & -0.03 & .23 & -0.07 & 0.02 & .17 & -0.02 & .27 & -0.07 & 0.02 & .32 \\
\hline
\end{tabular}

For primary analyses, a significance level of $\mathrm{p} \leq .01$ was used. All analyses are controlled for age, sex, and education. $B, p$, $C I$ refer only to the predictor of interest, $R^{2}$ indicates the amount of variance explained by the whole model, i.e., with all predictors included.

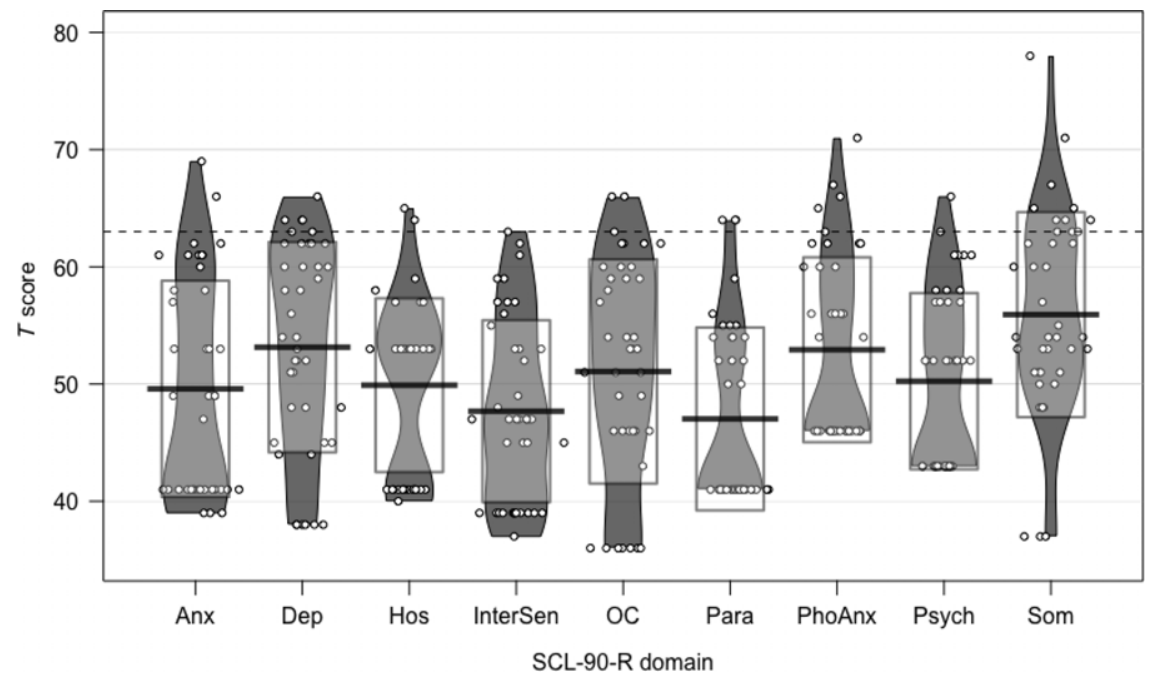

Figure 2. Pirateplot showing the distribution of psychiatric symptoms

The pirateplot reports $T$ scores of all individuals as separate data points. Noise (jitter) was added horizontally to reduce overlap among points with similar values. Anx = Anxiety subscale; Dep = Depression subscale; Hos = Hostility subscale; InterSen = Interpersonal sensibility subscale; $\mathrm{OC}=$ Obsessive-compulsive subscale (adjusted scores); Para = Paranoid ideation subscale; PhoAnx = Phobic anxiety subscale; Psych $=$ Psychoticism subscale; Som $=$ Somatization subscale. $T$ scores were used in order to indicate clinical symptom levels. Horizontal black lines indicate means. Standard deviation of the mean of each domain is indicated as transparent boxes. The dashed horizontal line indicates the clinical cutoff ( $T$ score $\geq 63$ ).

significantly better test results than men $(\mathrm{p}=.001)$ in both models with attention/executive function as a dependent variable.

\section{Subsample Analyses I: Relationship Between Psychiatric Symptoms and Performance-Based Cognitive Function}

The $T$ score distributions of psychiatric symptoms (SCL90-R) are shown in Figure 2. Most of the means are centered around a $T$ score of 50 (range: 47.0-55.9), with somatization having the highest mean and paranoid ideation the lowest. A maximum of 11 patients (26.8\% ; somatization) and a minimum of 1 (2.4\%; interpersonal sensitivity) scored above the clinical cutoff. The number of patients with clinical levels of obsessive-compulsive symptoms decreased from seven $(17.1 \%)$ to three $(7.3 \%)$ when an abbreviated obsessivecompulsive scale without the items assessing memory and concentration difficulties was used. None of the patients scored above cutoff on the total SCL-90-R score.

Table 4 displays the associations between psychiatric symptoms and performance-based cognitive function. Phobic anxiety was significantly negatively related to memory. Higher scores in the anxiety dimension (which focuses on symptoms of general anxiety like nervousness and trembling) and phobic anxiety dimension (which focuses on symptoms of agoraphobia) were related to lower attention/ executive function. Thus, two psychiatric domains had a significant negative relationship with performance-based attention/executive function, and one significant relationship between psychiatric symptoms and performance-based memory function was found. The SCL-90-R total score (both with the items measuring concentration and memory difficulties, and a modified score without these items included) was neither related to performance-based memory $(\mathrm{p}=.80$ and $\mathrm{p}=.83$, respectively) nor to attention/executive function $(\mathrm{p}=.11$ and $\mathrm{p}=.12$, respectively). 
Table 4. Subsample analyses I: associations between psychiatric symptoms and performance-based cognitive function ( $n=41)$

\begin{tabular}{|c|c|c|c|c|c|c|c|c|c|c|}
\hline \multirow[b]{3}{*}{ SCL-90-R domain } & \multicolumn{5}{|c|}{ Memory } & \multicolumn{5}{|c|}{ Attention/Executive function } \\
\hline & \multirow[b]{2}{*}{$B$} & \multirow[b]{2}{*}{$p$} & \multicolumn{2}{|c|}{$95 \% \mathrm{CI}$} & \multirow[b]{2}{*}{$R^{2}$} & \multirow[b]{2}{*}{$B$} & \multirow[b]{2}{*}{$p$} & \multicolumn{2}{|c|}{$95 \% \mathrm{CI}$} & \multirow[b]{2}{*}{$R^{2}$} \\
\hline & & & Lower & Upper & & & & Lower & Upper & \\
\hline Depression & -0.05 & .89 & -0.69 & 0.60 & .20 & -0.45 & .12 & -1.01 & 0.12 & .43 \\
\hline Anxiety & -0.44 & .23 & -1.18 & 0.30 & .23 & -0.70 & $.03 *$ & -1.34 & -0.06 & .47 \\
\hline Phobic anxiety ${ }^{\mathrm{b}}$ & -0.89 & $.05^{*}$ & -2.04 & -0.33 & .26 & -1.25 & $<.001 *$ & -1.72 & -0.17 & .51 \\
\hline Somatization & -0.27 & .23 & -0.77 & 0.23 & .23 & -0.42 & .06 & -0.85 & 0.02 & .45 \\
\hline Obsessive-compulsive & -0.03 & .93 & -0.59 & 0.54 & .20 & -0.29 & .24 & -0.79 & 0.21 & .42 \\
\hline Interpersonal sensitivity ${ }^{\mathrm{b}}$ & 0.29 & .64 & -0.98 & 1.15 & .21 & -0.31 & .27 & -0.96 & 0.13 & .40 \\
\hline Hostility $^{\mathrm{b}}$ & 1.36 & .06 & -0.76 & 2.26 & .33 & 0.05 & .92 & -0.91 & 0.99 & .39 \\
\hline Paranoid ideation $^{\mathrm{b}}$ & -0.48 & .08 & -1.06 & 0.38 & .24 & -0.05 & .86 & -0.68 & 0.47 & .39 \\
\hline Psychoticism & 0.43 & .56 & -1.04 & 1.89 & .21 & -0.38 & .57 & -1.70 & 0.94 & .39 \\
\hline
\end{tabular}

${ }^{\mathrm{b}}$ Bootstrapping is based on 5000 replicates, and bias-corrected-accelerated confidence intervals are shown. As the two items constituting the composite score for self-reported cognitive difficulties (item 9 and item 55 of the SCL-90-R) are a part of the obsessive-compulsive (OC) subscale, an adjusted OC score without these two items was calculated. For exploratory subsample analyses, a significance level of $\mathrm{p} \leq .05$ was applied. All analyses are controlled for age, sex, and education. $B, p, C I$ refer only to the predictor of interest, $R^{2}$ indicates the amount of variance explained by the whole model, i.e., with all predictors included.

Table 5. Frequencies of self-reported cognitive difficulties $(n=41)$

\begin{tabular}{lcc}
\hline \hline & Frequency & Percent \\
\hline Concentration difficulties $^{1}$ & 22 & $53.7 \%$ \\
Mild difficulties $^{1}$ & 18 & $43.9 \%$ \\
Moderate difficulties $^{2}$ & 2 & $4.9 \%$ \\
Severe difficulties $^{3}$ & 2 & $4.9 \%$ \\
Memory difficulties $^{1}$ & 32 & $78.0 \%$ \\
Mild difficulties $^{1}$ & 21 & $51.2 \%$ \\
Moderate difficulties $^{2}$ & 7 & $17.1 \%$ \\
Severe difficulties $^{3}$ & 4 & $9.7 \%$ \\
Difficulties in either memory or & 34 & $82.9 \%$ \\
concentration domain, or both $^{4}$ & & \\
\hline \hline
\end{tabular}

1"Mild difficulties" were defined as ratings (1) "a little bit" on the SCL-90-R. 2"Moderate difficulties" were defined as ratings (2) "moderately" on the SCL-90-R. "Severe difficulties" were defined as ratings (3) "quite a bit" and (4) "extremely". " "Difficulties in either memory or concentration domain, or both" was defined as ratings from (1) "a little bit" to (4) "extremely" in either the memory or the concentration domain, or both.

\section{Subsample Analyses II: Relationship Between Psychiatric Symptoms and Self-Reported Cognitive Function}

An overview of self-reported cognitive difficulties is displayed in Table 5.

Table 6 shows the associations between psychiatric symptoms and self-reported cognitive function. More self-reported cognitive difficulties were associated with higher symptom levels in all psychiatric domains $(\mathrm{p} \leq .05)$. Sex was significantly related to self-reported cognitive difficulties in the phobic anxiety $(\mathrm{p}=.05)$ and somatization model $(\mathrm{p}=.05)$. Neither age nor education was a significant predictor in any models. Both HADS-A and HADS-D were significantly positively associated with self-reported cognitive difficulties $(\mathrm{p} \leq .05)$. Higher education significantly predicted lower levels of self-reported cognitive difficulties in the HADSD-regression model $(\mathrm{p}=.05)$, but not in the HADS-A model. A higher SCL-90-R total score was related to more selfreported cognitive difficulties $(\mathrm{p} \leq .001)$.

\section{Correlations Between Self-Reported and Performance-Based Cognitive Function $(n=41)$}

Pearson correlations showed that more self-reported cognitive difficulties were related to lower performance-based attention/executive function as measured by the composite score. Also, all individual tests measuring attention/executive function, except TMT-A, were negatively correlated with self-reported cognitive difficulties. No significant association between self-reported cognitive difficulties and performancebased memory was found (Table 7).

\section{DISCUSSION}

\section{Principal Findings}

We studied the relationship of psychiatric symptoms with performance-based and self-reported cognitive function 3 months post-stroke. We found that depressive symptoms (HADS-D) were associated with performance-based memory difficulties in relatively well-functioning (based on baseline NIHSS, Barthel Index score, and MMSE) patients. Also, psychiatric symptoms were primarily related to self-reported rather than to performance-based cognitive difficulties: All nine psychiatric domains (anxiety, depression, phobic anxiety, obsessive-compulsive symptoms, somatization, hostility, interpersonal sensitivity, paranoid ideation, psychoticism) were negatively related to self-reported cognitive difficulties, whereas only two (anxiety, phobic anxiety) were associated with lower performance-based cognitive function. 
Table 6. Subsample analyses II: associations between psychiatric symptoms and self-reported cognitive function $(n=41)$

\begin{tabular}{|c|c|c|c|c|c|}
\hline \multirow[b]{3}{*}{ SCL-90-R domain } & \multicolumn{5}{|c|}{ Self-reported cognitive function } \\
\hline & \multirow[b]{2}{*}{$B$} & \multirow[b]{2}{*}{$p$} & \multicolumn{2}{|c|}{$95 \% \mathrm{CI}$} & \multirow[b]{2}{*}{$R^{2}$} \\
\hline & & & Lower & Upper & \\
\hline Depression & 1.08 & $<.001 *$ & 0.54 & 1.62 & .40 \\
\hline Anxiety & 0.94 & $.01 *$ & 0.24 & 1.63 & .28 \\
\hline Phobic anxiety ${ }^{\mathrm{b}}$ & 1.51 & $.02 *$ & 0.71 & 4.35 & .32 \\
\hline Somatization & 0.94 & $<.001 *$ & 0.53 & 1.34 & .46 \\
\hline Obsessive-compulsive & 1.06 & $<.001 *$ & 0.61 & 1.50 & .47 \\
\hline Interpersonal sensitivity & 1.20 & $<.01 *$ & 0.47 & 1.93 & .34 \\
\hline Hostility $^{\mathrm{b}}$ & 1.19 & $.01 *$ & 0.46 & 2.66 & .23 \\
\hline Paranoid ideation ${ }^{\mathrm{b}}$ & 1.01 & $.03 *$ & 0.41 & 2.62 & .29 \\
\hline \multirow[t]{2}{*}{ Psychoticism $^{\mathrm{b}}$} & 1.47 & $.01 *$ & 0.49 & 2.53 & .23 \\
\hline & & & \multicolumn{2}{|c|}{$95 \% \mathrm{CI}$} & \\
\hline HADS & $B$ & $p$ & Lower & Upper & $R^{2}$ \\
\hline HADS-D & 0.11 & $.03 *$ & 0.01 & 0.20 & .25 \\
\hline HADS-A & 0.08 & $.03 *$ & 0.01 & 0.15 & .24 \\
\hline
\end{tabular}

bootstrapping is based on 5000 replicates, and bias-corrected-accelerated confidence intervals are shown. As the two items constituting the composite score for self-reported cognitive difficulties (item 9 and item 55 of the SCL-90-R) are a part of the obsessive-compulsive subscale, an adjusted score without these two items was calculated. A significance level of $p \leq .05$ was applied. All analyses are controlled for age, sex, and education. $B, p, C I$ refer only to the predictor of interest, $R^{2}$ indicates the amount of variance explained by the whole model, i.e., with all predictors included.

Table 7. Correlations between performance-based and self-reported cognitive function $(n=41)$

\begin{tabular}{|c|c|c|c|c|c|c|c|c|c|c|c|}
\hline & \multicolumn{5}{|c|}{ Memory } & \multicolumn{6}{|c|}{ Attention/Executive function } \\
\hline & $\mathrm{CS}$ & REY-1 & REY-2 & RAVLT-1 & RAVLT- 2 & $\mathrm{CS}$ & TMT-A & TMT-B & CWIT-3 & CWIT-4 & Coding \\
\hline $\begin{array}{l}\text { Self-reported cognitive } \\
\text { function CS }\end{array}$ & -.25 & -.21 & -.18 & -.12 & -.17 & $-.40^{*}$ & -.24 & $-.42 * *$ & $-.33 *$ & $-.35^{*}$ & $-.36 *$ \\
\hline
\end{tabular}

$\mathrm{CS}=$ Composite score; ${ }^{*}$ Significant at $\mathrm{p} \leq .05 . * *$ Significant at $\mathrm{p} \leq .01$, two-tailed test. TMT-A/B and CWIT-3/4 scores have been multiplied with -1 so that a higher score indicates a better result.

\section{Primary Analyses: Relations of Symptoms of Anxiety and Depression to Performance-Based Cognitive Function}

Higher levels of depressive symptoms were associated with lower performance-based memory but not with attention/ executive function. Cognitive impairments and depression are highly prevalent after minor stroke (Moran et al., 2014), and several studies have found an association between symptoms of depression and cognitive domains such as verbal memory, cognitive speed (Barker-Collo, 2007), and executive function (Morsund et al., 2019). Our study extends these findings to patients with good outcomes after stroke. The patients included in our study were neither severely functionally impaired, nor had high levels of self-reported depression: With $13 \%$ having scores above cutoff on HADS-D, the prevalence of self-reported depression in our sample was substantially lower than commonly found in the post-acute phase after stroke (Hackett \& Pickles, 2014). This may partly be explained by the fact that our patients were assessed 3 months post-stroke, whereas the prevalence of depression seems to be higher during the first-month post-stroke compared to later stages (Kouwenhoven, Kirkevold, Engedal, \& Kim, 2011). Also, our sample consisted of $70 \%$ men, who are known to report less depressive symptoms (Poynter et al., 2009). However, the fact that depressive symptoms are not reported does not necessarily mean that they do not have them. Such underlying symptoms may still negatively influence cognitive function, and may therefore explain why men had lower scores on attention/executive function tests than women.

Our results indicate that stroke patients with good outcome can have underlying cognitive and emotional difficulties that may remain undetected if not specifically assessed. Whether depressive symptoms and poorer cognitive function influence each other, or whether they occur side by side, remains unclear. Some research suggests that selective serotonin reuptake inhibitors (SSRIs) improve executive function in stroke patients (Narushima, Paradiso, Moser, Jorge, \& Robinson, 2007), but divergent results on the effectiveness of pharmacotherapy to improve cognition post-stroke exist (Hackett, Anderson, \& House, 2005). Additionally, motivational interviewing has shown a promising effect on improving mood 
post-stroke (Cheng et al., 2015), and future studies should explore whether psychosocial and psychological interventions may improve cognitive function post-stroke.

Interestingly, HADS-A was not associated with performance-based measures, which differs from the findings of our subsample analyses when the SCL-90-R anxiety domain was used. Different anxiety measures may, at least in part, explain divergent results on the relationship between anxiety and cognitive function post-stroke.

\section{Subsample Analyses I: Psychiatric Symptoms and Performance-Based Cognitive Function}

With $2.4 \%-26.6 \%$ of patients above the clinical cutoff, levels of psychiatric symptoms were lower than usually found in this population (Ferro et al., 2016), possibly due to our patients having mild strokes without severe cognitive/ language problems. The somatization subdomain had the highest percentage of clinical cases $(26.8 \%)$, similar to other stroke samples (Rasquin et al., 2005). High scores may be due to physical impairment and pain post-stroke, and thus reflect actual physical health rather than somatization of underlying psychological distress.

Moreover, clinical levels of self-reported phobic anxiety were more common than clinical levels of self-reported generalized anxiety. This aligns with research showing that phobic anxiety is the most common anxiety subtype 3 months after a minor stroke (Chun, Whiteley, Dennis, Mead, \& Carson, 2018). The SCL-90-R phobic anxiety dimension measures symptoms associated with panic disorder with and without agoraphobia, which is defined as a persistent fear response to a specific person, place, object, or situation, leading to avoidance behavior (Derogatis, 1992). However, avoidance behavior in stroke patients may be due to poststroke sequelae such as motor impairment rather than symptoms of panic disorder. Thus, the phobic anxiety domain may in part capture self-reported motor symptoms instead of psychiatric distress. Nevertheless, how phobic anxiety impacts life post-stroke is an important avenue for future research, as phobic symptoms challenge the ability to live an ordinary life outside one's home and social participation.

We also found several cases $(17.1 \%)$ with obsessive-compulsive symptoms above the clinical cutoff. When using an abbreviated obsessive-compulsive-scale without the items assessing memory and concentration difficulties, the number of clinical cases decreased to three $(7.3 \%)$. This indicates that self-reported cognitive difficulties are a major contributor to elevated scores on this scale. With cognitive difficulties being common after cerebrovascular accidents, the SCL-90-R obsessive-compulsive subscale should be interpreted with caution in the stroke population.

Examining the association between psychiatric symptoms and performance-based function, we found that higher levels of anxiety and phobic anxiety were associated with poorer cognitive function. This differs from earlier work, which did not find a relation between anxiety and cognition
12 months after a minor stroke (Morsund et al., 2019). Inconsistent results might be due to the time of assessment, as anxiety is less prevalent 6-12 months post-stroke compared to the first 6 months (Knapp et al., 2020). Furthermore, inclusion criteria led to patients in the Morsund (2019) study being younger and less functionally impaired (NIHSS score) at baseline. Future studies with robust methodology are needed to further disentangle the relationship between post-stroke anxiety and performance-based cognitive function.

There were three additional interesting findings: First, only phobic anxiety was associated with both performancebased measures. Hence, phobic anxiety may not only be particularly prevalent post-stroke, but may also be uniquely associated with poorer test performance in a broader range of cognitive function.

Second, the SCL-90-R depression/anxiety scale and HADS were differently related to performance-based cognitive function. As in the full sample, HADS-D was negatively related to memory in the subsample, although nonsignificant. Thus, it is unlikely that divergent results are solely based on sample differences. Rather, low agreement between the SCL-90-R and HADS may be due to methodological differences. For example, some items of the SCL-90-R depression subscale (e.g., "Thoughts of ending my life.") do not have an equivalent in HADS-D, and single HADS items have shown low discrimination between anxiety and depression in stroke patients (Ayis, Ayerbe, Ashworth, \& Wolfe, 2018).

Third, the SCL-90-R total score was neither associated with performance-based attention/executive function, nor with memory. This indicates that the relationship of phobic anxiety and anxiety with cognitive function was not explained by overall psychopathological distress, but rather by specific symptom load in these domains.

\section{Subsample Analyses II: Psychiatric Symptoms and Self-Reported Cognitive Function}

Aligning with previous research, subjective cognitive difficulties were common, with around three-fourth stating memory difficulties, and about half of the patients reporting concentration difficulties. One possible explanation for the high prevalence of self-reported memory difficulties may be that patients with brain damage tend to interpret reduced processing speed, attention, and executive control as memory deficits (Lezak, Howieson, \& Loring, 2012).

When looking at the association between psychiatric symptoms and self-reported cognitive function, a clear picture emerged: All nine SCL-90-R domains were positively associated with subjective cognitive difficulties. This is consistent with studies that showed a relationship between depressive symptoms and self-reported cognitive difficulties in patients with mild cognitive impairment (Grambaite et al., 2013) and in stroke patients (van Rijsbergen et al., 2014). Our finding also aligns with reports of greater depression being 
associated with self-reported functional changes rather than performance-based cognitive changes in conditions such as HIV and hepatitis C (Bieliauskas et al., 2006).

While supporting previous findings, our study also provides a new insight: Our results indicate that the relationship between psychiatric symptoms and self-reported cognitive function may go far beyond depression and extend to a wide spectrum of psychiatric symptoms. Self-reported cognitive difficulties should, therefore, be in focus in clinical practice and research.

\section{Performance-Based and Self-Reported Cognitive Function}

Patients who reported more cognitive difficulties had significantly lower scores on four out of the five tests measuring attention/executive function, which may indicate that patients realistically evaluated their cognitive abilities in this domain.

However, none of the memory tests were associated with self-reported cognitive function. One possible explanation for this may be that patients face greater difficulties in their daily lives post-stroke than what memory results can indicate. Biases may also occur if patients with poorer memory simply do not remember having these difficulties and thus do not report them. Moreover, the lacking association between self-reported cognitive function and memory test results may be due to the items measuring self-rated cognitive function being rather nonspecific. For example, participants may have interpreted "Trouble remembering things" as difficulties with short-term/working memory or word-finding difficulties - domains that are not assessed with the memory tests used in this study.

Interestingly, our results differ from earlier work that has commonly found an association between self-reported cognitive difficulties and poorer performance-based memory, and not to lower function in other cognitive domains (van Rijsbergen, Mark, de Kort, \& Sitskoorn, 2014). However, other cognitive domains than memory were often not reported (van Rijsbergen, Mark, de Kort, \& Sitskoorn, 2014). Those few that reported results on the relation between subjective cognitive difficulties and performance-based attention/ executive function (but were nonsignificant), had different selection criteria and tested patients in the acute phase post-stroke (Duits, Munnecom, van Heugten, \& van Oostenbrugge, 2008), or studied specific aspects of self-rated cognition (Winkens, Van Heugten, Fasotti, \& Wade, 2009).

\section{Strengths, Limitations, and Future Directions}

Our study extends and substantiates prior research by including both performance-based and self-rated cognitive function as an outcome measure. By assessing a well-functioning stroke population with an extensive neuropsychological test battery, we gained important new insights for a group of patients with relatively good outcomes post-stroke, which are relevant for research and clinical practice.
We aimed to explore a broad spectrum of psychiatric symptoms and their relation to performance-based and self-reported cognitive function, which led to numerous regression models. This comes along with a greater risk for significant results accumulated by chance due to multiple testings. As little is known about the relation between psychiatric symptoms and cognitive function in high-functioning post-stroke populations, our subsample analyses were explorative, and we did not statistically adjust for multiple testing. The results of our subsample analyses should, therefore, be interpreted with caution and further studied with a larger sample size.

The sample size of our subsample analyses was small, limiting statistical power. However, the subsample for secondary aims was highly similar to those who were not included in subsample analyses on key demographic and clinical variables. We hope that our results, though preliminary, stimulate larger studies.

Furthermore, our patients were rather well-functioning, and generalizability of our findings to patients with lower functioning after moderate or severe stroke is therefore limited.

Processing speed, which we have not controlled for, is a major component in all neuropsychological tests used to measure attention/executive function in this study. Thus, results may be influenced by stroke sequelae such as mild motor impairment. Including tests into the composite that are not majorly dependent on processing speed would be an interesting enhancement of this study.

Also, using a summarized score for cognitive function makes it impossible to predict difficulties or abilities in a specific domain of performance-based cognitive function (e.g., delayed verbal recall). However, composite scores minimize possible ceiling effects, and we see it as the strength that a substantial number of neuropsychological tests were included in each composite.

Furthermore, no performance validity tests were included. However, all patients were encouraged to put forth their best efforts to perform at their best level of ability and informed that the results of the neuropsychological testing may be used to evaluate their driving abilities. There were also no apparent gains that would be contingent on lowered performance.

Moreover, there is no "gold standard" to define selfreported cognitive function post-stroke (van Rijsbergen, Mark, de Kort, \& Sitskoorn, 2014). We measured subjective cognitive difficulties with two items. Although this may not suffice to capture a differentiated picture, we assessed subjective memory and concentration difficulties, which are among the most commonly reported difficulties post-stroke (Lamb, Anderson, Saling, \& Dewey, 2013).

Future studies should take patients' premorbid cognitive function and comorbidities into account, which may influence test results independently from a stroke. Finally, including a control group without stroke to compare our results to those of a healthy, age-matched population, would be an important next step. 


\section{Clinical Implications}

This study provides new insights that may help to improve diagnosis and treatment for well-functioning stroke patients. First, stroke patients with self-reported cognitive difficulties in the post-acute period may be at a higher risk of psychiatric symptoms. Subjective cognitive difficulties post-stroke should be taken seriously, and clinicians should be made aware that they may not necessarily reflect actual cognitive impairment but psychiatric symptoms.

Second, an early screening of a broad spectrum of psychiatric symptoms post-stroke, and a more extensive follow-up for those at risk for higher levels of psychiatric distress, may help that patients get adequate treatment.

Third, as subjective cognitive difficulties may predict cognitive decline post-stroke (van Rijsbergen et al., 2014), clinicians should be alert if their patients report cognitive difficulties because these may signal patients in need of a more thorough follow-up.

Finally, different psychiatric symptoms were associated with lower performance-based cognitive function in wellfunctioning stroke patients. Clinicians should be aware that patients with good outcome and prognosis may have "hidden" symptoms that are hard to detect in general clinical assessments, but may still impact patients' life post-stroke.

\section{SUPPLEMENTARY MATERIAL}

To view supplementary material for this article, please visit https://doi.org/10.1017/S1355617721000187.

\section{ACKNOWLEDGMENTS}

The study is supported by grants from the South-Eastern and Central Norway Regional Health Authority. We wish to thank Per Selnes and John Hald for contributions to data collection, Jūrate Šaltyte Benth for statistical advice, and all participants for their time and effort.

\section{CONFLICTS OF INTEREST}

The authors have no conflicts of interests to declare.

\section{REFERENCES}

Aben, I., Verhey, F., Lousberg, R., Lodder, J., \& Honig, A. (2002). Validity of the beck depression inventory, hospital anxiety and depression scale, SCL-90, and Hamilton depression rating scale as screening instruments for depression in stroke patients. Psychosomatics, 43(5), 386-393. doi: 10.1176/appi.psy.43.5.386

Ayis, S.A., Ayerbe, L., Ashworth, M., \& Wolfe, D.A. (2018). Evaluation of the Hospital Anxiety and Depression Scale (HADS) in screening stroke patients for symptoms: Item Response Theory (IRT) analysis. Journal of Affective Disorders, 228, 33-40. doi: $10.1016 /$ j.jad.2017.11.037
Barker-Collo, S.L. (2007). Depression and anxiety 3 months post stroke: Prevalence and correlates. Archives of Clinical Neuropsychology, 22(4), 519-531. doi: 10.1016/j.acn.2007.03.002

Bieliauskas, L.A., Back-Madruga, C., Lindsay, K.L., Snow, K.K., Kronfol, Z., Lok, A.S., ... Fontana, R.J. (2006). Clinical relevance of cognitive scores in hepatitis $\mathrm{C}$ patients with advanced fibrosis. Journal of Clinical and Experimental Neuropsychology, 28(8), 1346-1361. doi: 10.1080/13803390500473720

Boone, K.B., Miller, B.L., Swartz, R., Lu, P., \& Lee, A. (2003). Relationship between positive and negative symptoms and neuropsychological scores in frontotemporal dementia and Alzheimer's disease. Journal of the International Neuropsychological Society, 9(5), 698-709. doi: 10.1017/S135561770395003x

Carlsson, G.E., Moller, A., \& Blomstrand, C. (2003). Consequences of mild stroke in persons $<75$ years - a 1-year follow-up. Cerebrovascular Diseases, 16(4), 383-388. doi: 10.1159/0000 72561

Carrozzino, D., Vassend, O., Bjorndal, F., Pignolo, C., Olsen, L.R., \& Bech, P. (2016). A clinimetric analysis of the Hopkins Symptom Checklist (SCL-90-R) in general population studies (Denmark, Norway, and Italy). Nordic Journal of Psychiatry, 70(5), 374-379. doi: 10.3109/08039488.2016.1155235

Cheng, D., Qu, Z., Huang, J., Xiao, Y., Luo, H., \& Wang, J. (2015). Motivational interviewing for improving recovery after stroke. Cochrane Database of Systematic Reviews (6). CD011398. doi: 10.1002/14651858.CD011398.pub2

Chun, H.Y., Whiteley, W.N., Dennis, M.S., Mead, G.E., \& Carson, A.J. (2018). Anxiety after stroke: The importance of subtyping. Stroke, 49(3), 556-564. doi: 10.1161/STROKEAHA.117.02 0078

Cumming, T.B., Marshall, R.S., \& Lazar, R.M. (2012). Stroke, cognitive deficits, and rehabilitation: Still an incomplete picture. International Journal of Stroke, 8(1), 38-45. doi: 10.1111/j. 1747-4949.2012.00972.x

Delis, D.C., Kaplan, E., \& Kramer, J.H. (2001). Delis and Kaplan D-KEFS Executive Functions System. San Antonio, TX: The Psychological Corporation.

Derogatis, L.R. (1992). SCL-90-R: Administration, Scoring \& Procedures Manual-II for the R(evised) Version and Other Instruments of the Psychopathology Rating Scale Series. Towson, MD: Clinical Psychometric Research.

Duits, A., Munnecom, T., van Heugten, C., \& van Oostenbrugge, R.J. (2008). Cognitive complaints in the early phase after stroke are not indicative of cognitive impairment. Journal of Neurology Neurosurgery and Psychiatry, 79(2), 143-146. doi: 10.1136/ jnnp.2007.114595

Ferro, J.M., Caeiro, L., \& Figueira, M.L. (2016). Neuropsychiatric sequelae of stroke. Nature Reviews Neurology, 12, 269. doi: 10. 1038/nrneurol.2016.46

Folstein, M.F., Folstein, S.E., \& McHugh, P.R. (1975). "Mini Mental State": A practical method for grading the cognitive state of patients for the clinician. Journal of Psychiatry Research, 12, 189-198.

Fure, B., Wyller, T.B., Engedal, K., \& Thommessen, B. (2006). Emotional symptoms in acute ischemic stroke. International Journal of Geriatric Psychiatry, 21(4), 382-387. doi: 10.1002/ gps. 1482

Gibbons, L.E., Carle, A.C., Mackin, R.S., Harvey, D., Mukherjee, S., Insel, P., ... Alzheimer's Disease Neuroimaging Initiative (2012). A composite score for executive functioning, validated in Alzheimer's Disease Neuroimaging Initiative (ADNI) participants with baseline mild cognitive 
impairment. Brain Imaging and Behavior, 6(4), 517-527. doi: 10.1007/s11682-012-9176-1

Grambaite, R., Hessen, E., Auning, E., Aarsland, D., Selnes, P., \& Fladby, T. (2013). Correlates of subjective and mild cognitive impairment: Depressive symptoms and CSF biomarkers. Dementia and Geriatric Cognitive Disorders Extra, 3(1), 291-300. doi: 10.1159/000354188

Grosdemange, A., Monfort, V., Richard, S., Toniolo, A.M., Ducrocq, X., \& Bolmont, B. (2015). Impact of anxiety on verbal and visuospatial working memory in patients with acute stroke without severe cognitive impairment. Journal of Neurology Neurosurgery and Psychiatry, 86(5), 513-519. doi: 10.1136/ jnnp-2014-308232

Hackett, M.L., Anderson, C.S., \& House, A.O. (2005). Management of depression after stroke. Stroke, 36(5), 1092-1097. doi:doi: 10.1161/01.STR.0000162391.27991.9d

Hackett, M.L., Kohler, S., O’Brien, J.T., \& Mead, G.E. (2014). Neuropsychiatric outcomes of stroke. Lancet Neurology, 13(5), 525-534. doi: 10.1016/S1474-4422(14)70016-X

Hackett, M.L. \& Pickles, K. (2014). Part I: Frequency of depression after stroke: An updated systematic review and meta-analysis of observational studies. International Journal of Stroke, 9(8), 1017-1025. doi: 10.1111/ijs.12357

IBM Corp. (2017). IBM SPSS Statistics for Windows, Version 25.0. Armonk, NY: IBM Corp.

Ihle-Hansen, H., Ihle-Hansen, H., Thommessen, B., Bruun Wyller, T., Engedal, K., Øksengård, A.R., .. . Fure, B. (2011). Incidence and subtypes of MCI and dementia 1 year after first-ever stroke in patients without pre-existing cognitive impairment. Dementia and Geriatric Cognitive Disorders, 32(6), 401-407. doi: 10.1159/ 000335361

Johnson, C.O., Nguyen, M., Roth, G.A., Nichols, E., Alam, T., Abate, D., ... Murray, C.J.L. (2019). Global, regional, and national burden of stroke, 1990-2016: A systematic analysis for the Global Burden of Disease Study 2016. The Lancet Neurology, 18(5), 439-458. doi: 10.1016/S1474-4422(19)30034-1

Kapoor, A., Lanctot, K. L., Bayley, M., Kiss, A., Herrmann, N., Murray, B. J., \& Swartz, R. H. (2017). "Good outcome" Isn't good enough cognitive impairment, depressive symptoms, and social restrictions in physically recovered stroke patients. Stroke, 48(6), 1688-1690. doi:10.1161/Strokeaha.117.016728

Knapp, P., Dunn-Roberts, A., Sahib, N., Cook, L., Astin, F., Kontou, E., \& Thomas, S.A. (2020). Frequency of anxiety after stroke: An updated systematic review and meta-analysis of observational studies. International Journal of Stroke, 15(3), 244-255. doi: $10.1177 / 1747493019896958$

Kouwenhoven, S.E., Kirkevold, M., Engedal, K., \& Kim, H.S. (2011). Depression in acute stroke: prevalence, dominant symptoms and associated factors. A systematic literature review. Disability and Rehabilitation, 33(7), 539-556. doi: 10.3109/ 09638288.2010.505997

Lamb, F., Anderson, J., Saling, M., \& Dewey, H. (2013). Predictors of subjective cognitive complaint in postacute older adult stroke patients. Archives of Physical Medicine and Rehabilitation, 94(9), 1747-1752. doi: 10.1016/j.apmr.2013.02.026

Leiknes, K.A., Dalsbø, T.K., \& Siqueland, J. (2016). Måleegenskaper ved den norske versjonen av Hospital Anxiety and Depression Scale (HADS). [Psychometric assessment of the Norwegian version of the Hospital Anxiety and Depression Scale (HADS). Retrieved from https://www.fhi.no/publ/2016/ maleegenskaper-ved-den-norske-versj/:
Lezak, M.D., Howieson, D.B., \& Loring, D.W. (2004). Neuropsychological Assessment (4th ed.). New York: Oxford University Press.

Loubinoux, I., Kronenberg, G., Endres, M., Schumann-Bard, P., Freret, T., Filipkowski, R.K., ... Popa-Wagner, A. (2012). Post-stroke depression: mechanisms, translation and therapy. Journal of Cellular and Molecular Medicine, 16(9), 1961-1969. doi: 10.1111/j.1582-4934.2012.01555.x

Mahoney, F.I. \& Barthel, D.W. (1965). Functional evaluation: The Barthel Index: A simple index of independence useful in scoring improvement in the rehabilitation of the chronically ill. Maryland State Medical Journal, 14, 61-65.

McKevitt, C., Fudge, N., Redfern, J., Sheldenkar, A., Crichton, S., Rudd, A.R., ... Wolfe, C.D.A. (2011). Self-reported long-term needs after stroke. Stroke, 42(5), 1398-1403. doi: 10.1161/ STROKEAHA.110.598839

Medeiros, G.C., Roy, D., Kontos, N., \& Beach, S.R. (2020). Poststroke depression: A 2020 updated review. General Hospital Psychiatry. 66, 70-80. doi: 10.1016/j.genhosppsych.2020.06.011

Meyers, J.E. \& Meyers, K.R. (1995). Rey Complex Figure Test and Recognition Trial (RCFT): Psychological Assessment Resources Odessa, FL.

Moran, G.M., Fletcher, B., Feltham, M.G., Calvert, M., Sackley, C., \& Marshall, T. (2014). Fatigue, psychological and cognitive impairment following transient ischaemic attack and minor stroke: a systematic review. European Journal of Neurology, 21(10), 1258-1267. doi: 10.1111/ene.12469

Morsund, A.H., Ellekjaer, H., Gramstad, A., Reiestad, M.T., Midgard, R., Sando, S.B., ... Naess, H. (2019). Cognitive and Emotional Impairment after Minor Stroke and NonST-Elevation Myocardial Infarction (NSTEMI): A prevalence study. Stroke Research and Treatment, 2019, 2527384. doi: 10. 1155/2019/2527384

Narushima, K., Paradiso, S., Moser, D.J., Jorge, R., \& Robinson, R.G. (2007). Effect of antidepressant therapy on executive function after stroke. British Journal of Psychiatry, 190(3), 260-265. doi: 10.1192/bjp.bp.106.025064

Nijsse, B., van Heugten, C.M., van Mierlo, M.L., Post, M.W., de Kort, P.L., \& Visser-Meily, J.M. (2017). Psychological factors are associated with subjective cognitive complaints 2 months post-stroke. Neuropsychological Rehabilitation, 27(1), 99-115. doi: 10.1080/09602011.2015.1065280

Poynter, B., Shuman, M., Diaz-Granados, N., Kapral, M., Grace, S.L., \& Stewart, D.E. (2009). Sex differences in the prevalence of post-stroke depression: A systematic review. Psychosomatics, 50(6), 563-569. doi: 10.1176/appi.psy.50.6.563

Rasquin, S., Lodder, J., \& Verhey, F. (2005). The association between psychiatric and cognitive symptoms after stroke: A prospective study. Cerebrovascular Diseases, 19(5), 309-316. doi: 10.1159/000084499

Reitan, R.M. \& Wolfson, D. (1985). The Halstead-Reitan Neuropsychological Test Battery. Tuscon, AZ: Neuropsychology Press.

Robinson, R. \& Jorge, R. (2016). Post-stroke depression: A review. American Journal of Psychiatry, 173(3), 221-231. doi: 10.1176/ appi.ajp.2015.15030363

Selnes, P., Grambaite, R., Rincon, M., Bjornerud, A., Gjerstad, L., Hessen, E., ... Fladby, T. (2015). Hippocampal complex atrophy in poststroke and mild cognitive impairment. Journal of Cerebral Blood Flow and Metabolism, 35(11), 1729-1737. doi: 10.1038/ jcbfm. 2015.110 
Skidmore, E.R., Whyte, E.M., Holm, M.B., Becker, J.T., Butters, M.A., Dew, M.A., ... Lenze, E.J. (2010). Cognitive and affective predictors of rehabilitation participation after stroke. Archives of Physical Medicine and Rehabilitation, 91(2), 203-207. doi: 10.1016/j.apmr.2009.10.026

Sun, J.-H., Tan, L., \& Yu, J.-T. (2014). Post-stroke cognitive impairment: epidemiology, mechanisms and management. Annals of Translational Medicine, 2(8), 80-80. doi: 10.3978/j.issn.23055839.2014.08.05

Terrill, A.L., Schwartz, J.K., \& Belagaje, S.R. (2018). Best practices for the interdisciplinary rehabilitation team: A review of mental health issues in mild stroke survivors. Stroke Research and Treatment, 2018, 6187328. doi: 10.1155/2018/6187328

van Rijsbergen, M.W., Mark, R.E., de Kort, P.L., \& Sitskoorn, M.M. (2014). Subjective cognitive complaints after stroke: A systematic review. Journal of Stroke and Cerebrovascular Diseases, 23(3), 408-420. doi: 10.1016/j.jstrokecerebrovasdis.2013.05.003

van Rijsbergen, M.W.A., Mark, R.E., Kop, W.J., de Kort, P.L.M., \& Sitskoorn, M.M. (2020). Course and predictors of subjective cognitive complaints during the first 12 months after stroke. Journal of Stroke and Cerebrovascular Diseases, 29(3), 104588. doi: 10. 1016/j.jstrokecerebrovasdis.2019.104588
Wechsler, D. (1999). Wechsler Abbreviated Scale of Intelligence (WASI). San Antonio, TX: The Psychological Corporation.

Wechsler, D. (2003). Wechsler Adult Intelligence Scale - Third Edition: Manual. San Antonio, TX: The Psychological Corporation.

Williams, L.S., Ghose, S.S., \& Swindle, R.W. (2004). Depression and other mental health diagnoses increase mortality risk after ischemic stroke. American Journal of Psychiatry, 161(6), 1090-1095. doi: 10.1176/appi.ajp.161.6.1090

Winkens, I., Van Heugten, C., Fasotti, L., \& Wade, D. (2009). Reliability and validity of two new instruments for measuring aspects of mental slowness in the daily lives of stroke patients. Neuropsychological Rehabilitation, 19(1), 64-85. doi: 10.1080/ 09602010801913650

Zigmond, A.S. \& Snaith, R.P. (1983). The hospital anxiety and depression scale. Acta Psychiatrica Scandinavica, 67(6), 361-370.

Zinn, S., Dudley, T.K., Bosworth, H.B., Hoenig, H.M., Duncan, P.W., \& Horner, R.D. (2004). The effect of poststroke cognitive impairment on rehabilitation process and functional outcome. Archives of Physical Medicine and Rehabilitation, 85(7), 1084-1090. doi: 10.1016/j.apmr.2003.10.022 